\title{
Stabilization of decentralized control systems by means of periodic feedback
}

\author{
Javad Lavaei ${ }^{\text {a }}$, Amir G. Aghdam ${ }^{\mathrm{b}}$ \\ ${ }^{a}$ Department of Control and Dynamical Systems, California Institute of Technology, Pasadena, CA 91125, USA \\ ${ }^{\mathrm{b}}$ Department of Electrical and Computer Engineering, Concordia University, Montréal, QC H3G 1M8, Canada
}

\begin{abstract}
This paper deals with structurally constrained periodic control design for interconnected systems. It is assumed that the system is linear time-invariant (LTI), observable and controllable, and that its modes are distinct and nonzero. It is shown that the notions of quotient fixed mode (QFM) and structured decentralized fixed mode (SDFM) are equivalent for this class of systems. Then, it is proved that if the system is decentrally stabilizable, then one candidate for the decentralized stabilizing controller is a time-varying one consisting of a decentralized LTI discrete-time compensator and a zero-order hold. More specifically, the non-quotient fixed modes of the system will be eliminated via sampling for almost all sampling periods, while any QFM will still remain a fixed mode. The results obtained are ultimately extended to the case when the system has some repeated modes, none of which is a DFM.
\end{abstract}

Key words: Decentralized control, Sampled-data systems, Time-varying controllers

\section{Introduction}

In control of large-scale interconnected systems, it is often desired to have some form of decentralization. In fact, for such systems it is not realistic to assume that all output measurements can be transmitted to every local control station. Problems of this kind appear, for example, in electric power systems, communication networks, large space structures, robotic systems, economic systems and traffic networks, to name only a few. Typical large-scale control systems have several local control stations, which observe only local outputs and control only local inputs. All the controllers are involved, however, in the overall operation of the control system. In the past three decades, the problem of decentralized control has been thoroughly investigated in the literature, and a variety of its characteristics are studied (Jamshidi, 1997; Siljak, 1991).

The notion of decentralized fixed modes (DFM) was introduced in Wang and Davison (1973) to identify those

\footnotetext{
‡ This work has been supported by the Natural Sciences and Engineering Research Council of Canada under grant RGPIN-262127-07.
}

Email addresses: lavaei@cds.caltech.edu (Javad Lavaei), aghdam@ece.concordia.ca (Amir G. Aghdam). modes of an interconnected system which are fixed with respect to any linear time-invariant (LTI) decentralized control law. In addition, structurally fixed modes were defined in Sezer and Siljak (1981), and it was shown that a mode is fixed due to either the structure of the system, or the perfect matching of its parameters. This idea was later used in Ozguner and Davison (1985) to classify the DFMs of a decoupled decentralized system as being either structured or unstructured. Moreover, it was shown in Ozguner and Davison (1985) that the distinct and nonzero unstructured DFMs of a system can be eliminated via sampling. It is noteworthy that the structurally fixed modes of a system are not invariant under similarity transformation and mainly depend on the realization of the system. In contrast, the structured or unstructured DFMs of a system are defined based on the decoupled model of the system, while their fundamental properties (such as movability by means of sampling) hold for any realization of the system.

As discussed above, the notion of a DFM was mainly introduced to investigate stabilizability of a system with respect to a decentralized LTI controller. In other words, it does not provide any result on the stabilizability of a system by means of a decentralized controller of any general type, i.e. nonlinear or time-varying. On the other hand, structured DFMs were basically defined to deter- 
mine whether or not a system is stabilizable by means of merely a sampled-data controller, as a special class of linear time-varying (LTV) controllers. Thus, this definition does not address the more general question of stabilizability with respect to a decentralized non-LTI controller.

The notion of quotient fixed modes (QFM) was introduced in Gong and Aldeen (1997) based on the graph topology of the interconnected system, to identify the modes that remain fixed with respect to a general nonlinear or time-varying decentralized controller. This notion provides a key result on the characterization of DFMs. It was also shown in Gong and Aldeen (1997) that a decentralized control system can be stabilized with any arbitrary degree of exponential stability if and only if it has no QFMs.

This paper investigates the decentralized control design problem for the controllable and observable finitedimensional LTI systems with distinct and nonzero modes. It is shown that for this broad class of systems, the notions of QFM and structured DFM are, in fact, the same. Using this result, it is proved that if a system (with said properties) is decentrally stabilizable under a general control law, then there exists a decentralized LTI discrete-time controller with a simple zero-order hold $(\mathrm{ZOH})$ to stabilize it. This results from the fact that the non-quotient DFMs of the continuous-time system are no longer fixed in the sampled system. It is noteworthy that this result can be considered as a generalization of the ones presented in Sezer and Siljak (1990), but for single-rate linear systems. Furthermore, some important properties of structured DFMs are investigated, which lead to a simple approach to determine the QFMs of a system. A numerical example is given to clarify the main results of the paper step by step.

This paper is organized as follows. First, some of the existing results and notions are reviewed in Section 2, which are exploited to develop the main results of the paper in Section 3. A numerical example is provided in this section, which is further examined in Section 4 to elucidate the ideas of the present work. Finally, some concluding remarks are presented in Section 5.

\section{Preliminaries}

Consider an LTI interconnected system $\mathcal{S}$ consisting of $\nu$ subsystems $S_{1}, S_{2}, \ldots, S_{\nu}$, and suppose that its modes are all distinct and nonzero (this condition is assumed to hold throughout the paper, unless stated otherwise). One can employ a proper similarity transformation to decouple the state-space model of the system. Let the resultant model be given by:

$$
\begin{aligned}
& \dot{x}(t)=A x(t)+\sum_{i=1}^{\nu} B_{i} u_{i}(t) \\
& y_{i}(t)=C_{i} x(t), \quad i \in \bar{\nu}:=\{1,2, \ldots, \nu\}
\end{aligned}
$$

where $x(t) \in \Re^{n}$ is the state, and $u_{i}(t) \in \Re^{m_{i}}$ and $y_{i}(t) \in$ $\Re^{r_{i}}$ are the input and the output of the $i$-th subsystem, respectively, and

$$
A=\operatorname{diag}\left(\left[\sigma_{1}, \sigma_{2}, \ldots, \sigma_{n}\right]\right)
$$

(note that by assumption $\sigma_{i} \neq 0, \sigma_{i} \neq \sigma_{j}, \forall i, j \in$ $\{1,2, \ldots, n\}, i \neq j)$. To avoid triviality, assume also that the system $\mathcal{S}$ is controllable and observable. Define now:

$$
\begin{aligned}
B & :=\left[\begin{array}{llll}
B_{1} & B_{2} & \cdots & B_{\nu}
\end{array}\right] \\
C & :=\left[\begin{array}{llll}
C_{1}^{T} & C_{2}^{T} & \cdots & C_{\nu}^{T}
\end{array}\right]^{T}, \\
u(t) & :=\left[\begin{array}{llll}
u_{1}(t)^{T} & u_{2}(t)^{T} & \cdots & u_{\nu}(t)^{T}
\end{array}\right]^{T}, \\
y(t) & :=\left[\begin{array}{llll}
y_{1}(t)^{T} & y_{2}(t)^{T} & \cdots & y_{\nu}(t)^{T}
\end{array}\right]^{T}, \\
m & :=\sum_{i=1}^{\nu} m_{i}, \quad r:=\sum_{i=1}^{\nu} r_{i}
\end{aligned}
$$

Decentralized control is referred to the set of local controllers, where each local controller is aimed to generate the input of its corresponding subsystem in terms of only the output of the same subsystem. In order to specify the local subsystems associated with the local controllers, the subsystems are enclosed within parentheses throughout the paper, if necessary. For instance, a decentralized controller for the system $\mathcal{S}\left(S_{1}, S_{2}, S_{3}\right)$ is the union of the local controllers $u_{i}(t)=g_{i}\left(y_{i}(t), t\right), i \in\{1,2,3\}$, corresponding to the subsystems $S_{1}, S_{2}, S_{3}$. Some important notions regarding different types of fixed modes will be outlined next, which are essential for developing the main results of the paper.

Definition 1 (Wang and Davison (1973)) The mode $\lambda \in \operatorname{sp}(A)$ is said to be a decentralized fixed mode (DFM) of the system $\mathcal{S}$, if it remains a mode of the closed-loop system under any arbitrary decentralized static feedback. In other words, $\lambda \in \operatorname{sp}(A)$ is a DFM of the system $\mathcal{S}$ if:

$$
\lambda \in \operatorname{sp}\left(A+\sum_{i=1}^{\nu} B_{i} K_{i} C_{i}\right), \quad \forall K_{i} \in \Re^{m_{i} \times r_{i}}, i \in \bar{\nu}
$$

It can be shown that a DFM is fixed with respect to not only static decentralized controllers (by definition) but also any arbitrary dynamic LTI decentralized controller. However, it is interesting to note that a proper non-LTI controller can eliminate certain types of DFMs (Gong and Aldeen, 1997; Anderson and Moore, 1981; Ozguner 
and Davison, 1985). In other words, a DFM is not necessarily fixed with respect to a time-varying or nonlinear decentralized control law.

Definition 2 Assume that $\lambda \in \operatorname{sp}(A)$ is a DFM of the system $\mathcal{S}\left(S_{1}, S_{2}, \ldots, S_{\nu}\right)$ given by $(1)$. The mode $\lambda$ is defined to be a structured decentralized fixed mode (SDFM) of the system, if it is a DFM of any system obtained from $\mathcal{S}$ by arbitrarily perturbing the nonzero entries of its matrices $B$ and $C$.

It is to be noted that in the definition of SDFM given in Ozguner and Davison (1985), the nonzero elements of $A$ (the elements on the main diagonal) are also assumed to be perturbed. Nonetheless, it can be easily verified that this assumption is not necessary, and hence can be simply relaxed.

Definition 3 Assume that $\lambda$ is a DFM of the system $\mathcal{S}\left(S_{1}, S_{2}, \ldots, S_{\nu}\right)$. Then, $\lambda$ is called an unstructured decentralized fixed mode (UDFM) if it is not an SDFM of $\mathcal{S}\left(S_{1}, S_{2}, \ldots, S_{\nu}\right)$. In other words, UDFMs originate from an exact matching of the system parameters, and any perturbation in the nonzero entries of the system matrices $A, B$ and $C$ would shift this type of modes (note that Definition 3 is not a trivial extension of Definition 2).

In order to illustrate the concepts of SDFM and UDFM, consider a system $\mathcal{S}$ consisting of three single-input single-output (SISO) subsystems with the following parameters:

$$
\begin{aligned}
A & =\operatorname{diag}([1,-2,-3]), \\
B_{1} & =\left[\begin{array}{lll}
0 & 0 & -1
\end{array}\right]^{T}, B_{2}=\left[\begin{array}{lll}
1 & 1 & 2
\end{array}\right]^{T}, B_{3}=\left[\begin{array}{lll}
2 & 1 & 5
\end{array}\right]^{T}, \\
C_{1} & =\left[\begin{array}{lll}
5 & 3 & 2
\end{array}\right], C_{2}=\left[\begin{array}{lll}
0 & -1 & 0
\end{array}\right], C_{3}=\left[\begin{array}{lll}
0 & -2 & 0
\end{array}\right]
\end{aligned}
$$

It can be easily verified that if the nonzero entries of the vectors $B_{i}$ and $C_{i}, \forall i \in\{1,2,3\}$, are replaced by any arbitrary numbers, the resultant system $\mathcal{S}$ still has a DFM at $\lambda=1$. In other words, this mode is immovable with respect to LTI decentralized controllers in all of the following systems:

$$
\begin{aligned}
A & =\operatorname{diag}([1,-2,-3]), \\
B_{1} & =\left[\begin{array}{lll}
0 & 0 & *
\end{array}\right]^{T}, B_{2}=\left[\begin{array}{lll}
* & * & *
\end{array}\right]^{T}, B_{3}=\left[\begin{array}{lll}
* & * & *
\end{array}\right]^{T}, \\
C_{1} & =\left[\begin{array}{lll}
* & * & *
\end{array}\right], C_{2}=\left[\begin{array}{lll}
0 & * & 0
\end{array}\right], C_{3}=\left[\begin{array}{lll}
0 & * & 0
\end{array}\right]
\end{aligned}
$$

where the $\mathrm{symbol} *$ represents the entries which can take any arbitrary values. This means that $\lambda=1$ is an SDFM of the system $\mathcal{S}$. Now, let the parameters $B_{1}, C_{2}$ and $C_{3}$ of the system $\mathcal{S}$ in (5) be replaced by the following:

$$
\begin{aligned}
& B_{1}=\left[\begin{array}{lll}
0 & 3 & -4
\end{array}\right]^{T}, \quad C_{2}=\left[\begin{array}{lll}
0 & -1 & -1
\end{array}\right], \\
& C_{3}=\left[\begin{array}{lll}
0 & -2 & -2
\end{array}\right]
\end{aligned}
$$

It is straightforward to show that $\lambda=1$ is a DFM of the system in this case too. However, if for example the second entry of $C_{2}$ is replaced by any value other than -1 , this mode will no longer be a DFM of the resultant system. As a result, $\lambda=1$ is a UDFM of the system $\mathcal{S}$. Note that this DFM is resulted from the exact matching of the nonzero entries of the matrices $A, B_{i}$ and $C_{i}, \forall i \in$ $\{1,2,3\}$ as pointed out in Definition 3 , and hence it is no longer fixed after perturbing the nonzero parameters of the system.

Define $\mathcal{S}_{d}$ as the discrete-time equivalent model of $\mathcal{S}$, obtained by using a constant sampling period $h>0$ and a zero-order hold $(\mathrm{ZOH})$. Thus, the state-space representation of $\mathcal{S}_{d}$ is as follows:

$$
\begin{gathered}
x[\kappa+1]=\bar{A} x[\kappa]+\sum_{i=1}^{\nu} \bar{B}_{i} u_{i}[\kappa] \\
y_{i}[\kappa]=C_{i} x[\kappa], \quad i \in \bar{\nu}
\end{gathered}
$$

where the discrete argument corresponding to the samples of any signal is enclosed in brackets (e.g., $x[\kappa]=$ $x(\kappa h))$. It can be easily shown that $\bar{A}=e^{A h}$, and $\bar{B}_{i}=$ $A^{-1}\left(\bar{A}-I_{n}\right) B_{i}, i \in \bar{\nu}$, where $I_{n}$ represents the $n \times n$ identity matrix (note that $A$ is invertible because by assumption its eigenvalues are all nonzero). Denote the subsystems of $\mathcal{S}_{d}$ corresponding to $S_{1}, S_{2}, \ldots, S_{\nu}$ with $\mathcal{S}_{d_{1}}, \mathcal{S}_{d_{2}}, \ldots, \mathcal{S}_{d_{\nu}}$. The following lemma is borrowed from Ozguner and Davison (1985).

Lemma 1 Assume that the system $\mathcal{S}\left(S_{1}, S_{2}, \ldots, S_{\nu}\right)$ has $P_{u}$ unstructured and $P_{s}$ structured DFMs, and denoted its SDFMs with $\lambda_{i}, i=1,2, \ldots, P_{s}$. The discrete-time system $\mathcal{S}_{d}\left(\mathcal{S}_{d_{1}}, \mathcal{S}_{d_{2}}, \ldots, \mathcal{S}_{d_{\nu}}\right)$ comprises $P_{s}$ structured DFMs $e^{\lambda_{i} h}, i=1,2, \ldots, P_{s}$ and no UDFMs, for almost all values of $h$.

Remark 1 The term "for almost all" in Lemma 1 means that the sampling periods for which the discrete-time system $\mathcal{S}_{d}\left(\mathcal{S}_{d_{1}}, \mathcal{S}_{d_{2}}, \ldots, \mathcal{S}_{d_{\nu}}\right)$ has UDFMs, either lie on a hypersurface in the one dimensional space (implying that the number of such sampling periods is finite), or are the pathological sampling periods which can potentially make the discrete-time system unobservable or uncontrollable (for the definition of a hypersurface see Davison and Wang (1973), and for the definition of pathological sampling refer to Chen and Francis (1995)).

Lemma 1 states that the UDFMs of the system $\mathcal{S}\left(S_{1}, S_{2}, \ldots, S_{\nu}\right)$ can (almost always) be displaced in the sampled model, whereas SDFMs still remain fixed after sampling. The questions arise as how to identify the type of DFMs, and how to design a stabilizing controller for a system with SDFMs (if possible). Note that a procedure is proposed in Ozguner and Davison (1985) to determine the type of the DFMs of the systems consisting of only two SISO subsystems.

On the other hand, the notion of quotient fixed modes (QFM) was introduced in Gong and Aldeen (1997) to 
investigate the stabilizability of interconnected systems under a general decentralized control law (i.e. nonlinear or time-varying). Since the definition of QFM is essential in the development of the main results of this paper, it is explained in the next two definitions.

Definition 4 Define the structural graph of the system $\mathcal{S}$ as a digraph with $\nu$ vertices which has a directed edge from the $i$-th vertex to the $j$-th vertex if and only if $C_{j}(s I-$ $A)^{-1} B_{i} \neq 0$, for any $i, j \in \bar{\nu}$. The structural graph of the system $\mathcal{S}$ is denoted by $\mathcal{G}$.

Partition $\mathcal{G}$ into the minimum number of strongly connected subgraphs denoted by $G_{1}, G_{2}, \ldots, G_{l}$ (recall that a digraph is strongly connected if and only if there exists a directed path from any vertex to any other vertices of the graph (Gong and Aldeen, 1997; Anderson and Moore, 1981)). Define the subsystem $\tilde{S}_{i}, i=1,2, \ldots, l$, as the union of all subsystems of $\mathcal{S}$ corresponding to the vertices in the subgraph $G_{i}$ (note that vertex $j$ in the graph $\mathcal{G}$ represents the subsystem $S_{j}$, for any $j \in \bar{\nu}$ ).

Definition $5 \lambda$ is said to be a QFM of the system $\mathcal{S}\left(S_{1}, S_{2}, \ldots, S_{\nu}\right)$, if it is a DFM of the system $\mathcal{S}\left(\tilde{S}_{1}, \tilde{S}_{2}, \ldots, \tilde{S}_{l}\right)$.

In order to cast light on the definition of a QFM, consider again the system $\mathcal{S}$ with the parameters given in (5). The transfer function matrix of this system is equal to:

$$
\left[\begin{array}{ccc}
\frac{-2}{s+3} & \frac{12 s^{2}+35 s+13}{(s-1)(s+2)(s+3)} & \frac{23 s^{2}+66 s+31}{(s-1)(s+2)(s+3)} \\
0 & \frac{-1}{s+2} & \frac{-1}{s+2} \\
0 & \frac{-2}{s+2} & \frac{-2}{s+2}
\end{array}\right]
$$

Hence, the structural graph of the system $\mathcal{S}$ is composed of two strongly connected subgraphs corresponding to vertex 1 (as the first subgraph), and vertices 2,3 (as the second subgraph). Therefore, the new subsystem $\tilde{S}_{1}$ is defined to be the subsystem $S_{1}$, while $\tilde{S}_{2}$ is the union of $S_{2}$ and $S_{3}$. The union of the subsystems $\tilde{S}_{1}$ and $\tilde{S}_{2}$ is sometimes referred to as a quotient system (Gong and Aldeen, 1997). It is imperative to note that:

- The DFMs of the system $\mathcal{S}\left(S_{1}, S_{2}, S_{3}\right)$ are also the modes of the closed-loop system in Figure 1, for any arbitrary LTI controllers $K_{1}, K_{2}$ and $K_{3}$. It can be easily verified that $\lambda=1$ is the only DFM of the system $\mathcal{S}$ given by $(5)$.

- The QFMs of the system $\mathcal{S}\left(S_{1}, S_{2}, S_{3}\right)$ are defined to be the DFMs of the system $\mathcal{S}\left(\tilde{S}_{1}, \tilde{S}_{2}\right)$, i.e., the fixed modes of the closed-loop system shown in Figure 2, for any arbitrary LTI controllers $\tilde{K}_{1}$ and $\tilde{K}_{2}$. For instance, it is easy to show that $\lambda=1$ is a QFM of the system $\mathcal{S}$ given by (5).

Lemma 2 (Gong and Aldeen (1997)) There exists a decentralized controller to stabilize the system $\mathcal{S}\left(S_{1}\right.$,

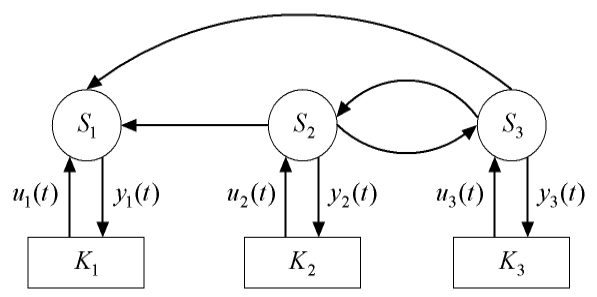

Fig. 1. The schematic of the decentralized control system $\mathcal{S}$ used for obtaining the DFMs.

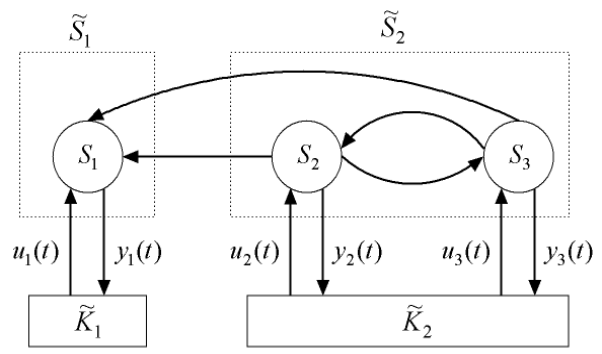

Fig. 2. The schematic of the decentralized control system $\mathcal{S}$ used for obtaining the QFMs.

$\left.S_{2}, \ldots, S_{\nu}\right)$ if and only if it does not have any $Q F M$ with a nonnegative real part.

Based on the developments in Anderson and Moore (1981), it is also shown in Gong and Aldeen (1997) that if the system is decentrally stabilizable, then a candidate stabilizing controller has the following LTV form:

$$
\begin{aligned}
& \dot{z}_{i}(t)=P_{1}^{i}(t) z_{i}(t)+P_{2}^{i}(t) y_{i}(t) \\
& u_{i}(t)=P_{3}^{i}(t) x_{i}(t)+P_{4}^{i}(t) y_{i}(t), \quad i \in \bar{\nu}
\end{aligned}
$$

where the matrices $P_{j}^{i}(t), j \in\{1,2,3,4\}, i \in \bar{\nu}$, are obtained from a number of observer-based controllers for some LTV augmented systems. As an alternative, the work Juan and Kabamba (1989) proposes a simpler class of LTV controllers, which can eliminate the non-QFMs of the system. It is worth mentioning that the results of Anderson and Moore (1981) leading to the controller (10) is extended in Khargonekar and Ozguler (1994) to periodically time-varying systems, which illuminates the necessity of having no QFMs for stabilization via periodic controllers.

The objective of this work is to show that another candidate decentralized stabilizing controller is a sampleddata control law, which can be obtained in a much simpler manner compared to the previously proposed LTV controllers. This will be addressed in the subsequent section.

\section{Effect of sampling on DFMs}

It is desired first to present some formulas for attaining the SDFMs of the system $\mathcal{S}$. To do so, the following 
notation is required.

Notation 1 For any $i \in \bar{\nu}$ :

- Denote the $\left(j_{1}, j_{2}\right)$ entry of $B_{i}$ with $b_{i}^{j_{1}, j_{2}}$, for any $1 \leq j_{1} \leq n, 1 \leq j_{2} \leq m_{i}$.

- Denote the $\left(j_{1}, j_{2}\right)$ entry of $C_{i}$ with $c_{i}^{j_{1}, j_{2}}$, for any $1 \leq j_{1} \leq r_{i}, 1 \leq j_{2} \leq n$.

In the case when there are only two SISO subsystems (i.e. $\nu=2, m_{1}=m_{2}=r_{1}=r_{2}=1$ ), it is shown in Ozguner and Davison (1985) that $\sigma_{i}$ is an SDFM of the system $\mathcal{S}$ if and only if either one of the conditions given below holds:

i) $b_{1}^{i, 1}=c_{2}^{1, i}=0, \quad b_{1}^{j, 1} c_{2}^{1, j}=0, \quad \forall j \in\{1,2, \ldots, i-1, i+$ $1, \ldots, n\}$.

ii) $b_{2}^{i, 1}=c_{1}^{1, i}=0, \quad b_{2}^{j, 1} c_{1}^{1, j}=0, \quad \forall j \in\{1,2, \ldots, i-1, i+$ $1, \ldots, n\}$.

In what follows, these simple formulas will be extended to general interconnected systems.

Theorem 1 The mode $\sigma_{i}, i \in\{1,2, \ldots, n\}$, is an $S D F M$ of the system $\mathcal{S}\left(S_{1}, S_{2}, \ldots, S_{\nu}\right), \nu \geq 2$, if and only if there exists a permutation of $\{1,2, \ldots, \nu\}$ denoted by distinct integers $i_{1}, i_{2}, \ldots, i_{\nu}$, as well as an integer $p$ between 1 and $\nu-1$, such that $b_{j_{1}}^{i, \alpha}=c_{j_{2}}^{\beta, i}=0$ and $b_{j_{1}}^{\mu, \alpha} c_{j_{2}}^{\beta, \mu}=0$, for all $j_{1}, j_{2}, \alpha, \beta$ and $\mu$ given by:

$$
\begin{aligned}
j_{1} & \in\left\{i_{1}, i_{2}, \ldots, i_{p}\right\}, \quad j_{2} \in\left\{i_{p+1}, i_{p+2}, \ldots, i_{\nu}\right\} \\
\alpha & \in\left\{1,2, \ldots, m_{j_{1}}\right\}, \quad \beta \in\left\{1,2, \ldots, r_{j_{2}}\right\} \\
\mu & \in \bar{\nu}-\{i\}
\end{aligned}
$$

(note that $\bar{\nu}-\{i\}$ is the set $\bar{\nu}$ without the entry $i$ ).

Remark 2 The formulas given in Theorem 1 state that $\sigma_{i}$ is an SDFM of the system $S$ if and only if one can partition the set $\{1,2, \ldots, \nu\}$ into two sets $\left\{i_{1}, i_{2}, \ldots, i_{p}\right\}$ and $\left\{i_{p+1}, i_{p+2}, \ldots, i_{\nu}\right\}$ such that the matrices $M_{1}$ and $M_{2}$ defined as:

$$
\begin{aligned}
& M_{1}=\left[\begin{array}{llll}
B_{i_{1}} & B_{i_{2}} & \cdots & B_{i_{p}}
\end{array}\right] \\
& M_{2}=\left[\begin{array}{llll}
C_{i_{p+1}}^{T} & C_{i_{p+2}}^{T} & \cdots & C_{i_{\nu}}^{T}
\end{array}\right]^{T}
\end{aligned}
$$

have the following properties:

a) The $i$-th row of the matrix $M_{1}$ and the $i$-th column of the matrix $M_{2}$ are both equal to zero vectors.

b) The product of any two entries in the $j$-th row of $M_{1}$ and the $j$-th column of $M_{2}$ is zero for all $j \in\{1,2, \ldots, i-$ $1, i+1, \ldots, \nu\}$.

Proof of Theorem 1: It is known that $\sigma_{i}$ is a DFM of the system $\mathcal{S}\left(S_{1}, S_{2}, \ldots, S_{\nu}\right)$ if and only if there exists a permutation of $\{1,2, \ldots, \nu\}$ denoted by distinct integers $i_{1}, i_{2}, \ldots, i_{\nu}$, as well as an integer $p$ between 0 and $\nu$, such that the rank of the following matrix is less than $n$ (Davison and Chang, 1990):

$$
\left[\begin{array}{cccc}
A-\sigma_{i} I_{n} & B_{i_{1}} & \ldots & B_{i_{p}} \\
C_{i_{p+1}} & 0 & \ldots & 0 \\
\vdots & \vdots & \ddots & \vdots \\
C_{i_{\nu}} & 0 & \ldots & 0
\end{array}\right]
$$

(note that 0 in the above matrix represents a zero block entry of proper dimension). In addition, since it is assumed that the system $\mathcal{S}$ is controllable and observable, the rank of the matrix (13) is equal to $n$ for $p=0$ and $p=\nu$ (note that the matrix (13) turns out to be the observability matrix for $p=0$ and the controllability matrix for $p=\nu)$. Therefore, the condition $0 \leq p \leq \nu$ given above can be replaced by $1 \leq p \leq \nu-1$. It is clear that the rank of the matrix $A-\sigma_{i} I_{n}$ is $n-1$, and, in addition, the $i$-th column and the $i$-th row of this matrix are both zero vectors. Hence, the rank of the matrix given in (13) is at least $n$, if and only if there exists a nonzero entry in either its $i$-th column or its $i$-th row. As a result, the rank of the matrix in (13) is less than $n$ if and only if both of the following conditions hold:

i) All of the entries of the $i$-th column and the $i$-th row of the matrix given in (13) are zero, i.e. $b_{j_{1}}^{i, \alpha}=c_{j_{2}}^{\beta, i}=0$ for any $\alpha, \beta, j_{1}$, and $j_{2}$ satisfying (11).

ii) The rank of the following matrix (which is a submatrix of the one given by (13)) is less than $n$ :

$$
\left[\begin{array}{ccccccc}
\sigma_{1}^{i} & \ldots & 0 & 0 & \ldots & 0 & b_{j_{1}}^{1, \alpha} \\
\vdots & \ddots & \vdots & \vdots & \ddots & \vdots & \vdots \\
0 & \ldots & \sigma_{i-1}^{i} & 0 & \ldots & 0 & b_{j_{1}}^{i-1, \alpha} \\
0 & \ldots & 0 & \sigma_{i+1}^{i} & \ldots & 0 & b_{j_{1}}^{i+1, \alpha} \\
\vdots & \ddots & \vdots & \vdots & \ddots & \vdots & \vdots \\
0 & \ldots & 0 & 0 & \ldots & \sigma_{n}^{i} & b_{j_{1}}^{n, \alpha} \\
c_{j_{2}}^{\beta, 1} & \ldots & c_{j_{2}}^{\beta, i-1} & c_{j_{2}}^{\beta, i+1} & \ldots & c_{j_{2}}^{\beta, n} & 0
\end{array}\right]
$$

for any $\alpha, \beta, j_{1}$, and $j_{2}$ satisfying (11), where $\sigma_{j}^{i}:=$ $\sigma_{j}-\sigma_{i}, i, j \in\{1,2, \ldots, n\}$. Partition the matrix given by (14) into four sub-matrices, and denote it with $\left[\begin{array}{cc}A_{i} & \Phi_{1} \\ \Phi_{2} & 0\end{array}\right]$, where $A_{i} \in \Re^{(n-1) \times(n-1)}, \Phi_{1} \in \Re^{(n-1) \times 1}$, and $\Phi_{2} \in \Re^{1 \times(n-1)}$. Since the matrix $A_{i}$ is nonsingular (because it is assumed that $\sigma_{1}, \ldots, \sigma_{n}$ are distinct), 
one can write:

$$
\operatorname{det}\left[\begin{array}{cc}
A_{i} & \Phi_{1} \\
\Phi_{2} & 0
\end{array}\right]=-\operatorname{det}\left(A_{i}\right) \times \operatorname{det}\left(\Phi_{2} A_{i}^{-1} \Phi_{1}\right)
$$

Thus, the rank of the matrix given in (14) is less than $n$ if and only if the scalar $\Phi_{2} A_{i}^{-1} \Phi_{1}$ is equal to 0 .

It can be concluded from Definition 2 and the above discussion that $\sigma_{i}$ is an SDFM if and only if condition (i) and the equality:

$$
\sum_{\mu=1, \mu \neq i}^{n} \frac{\bar{b}_{j_{1}}^{\mu, \alpha} \bar{c}_{j_{2}}^{\beta, \mu}}{\sigma_{\mu}^{i}}=0
$$

both hold, where $\bar{b}_{j_{1}}^{\mu, \alpha}$ and $\bar{c}_{j_{2}}^{\beta, \mu}$ represent any arbitrary nonzero multiples of $b_{j_{1}}^{\mu, \alpha}$ and $c_{j_{2}}^{\beta, \mu}$, respectively, for $\mu=$ $1,2, \ldots, n$. This condition is equivalent to the equality $b_{j_{1}}^{\mu, \alpha} c_{j_{2}}^{\beta, \mu}=0$ for $\mu=1,2, \ldots, n, \mu \neq i$.

Corollary 1 Assume that $\sigma_{i}, i \in\{1,2, \ldots, n\}$, is an $S D F M$ of the system $\mathcal{S}\left(S_{1}, S_{2}, \ldots, S_{\nu}\right)$. There exists a permutation of $\{1,2, \ldots, \nu\}$ denoted by distinct integers $i_{1}, i_{2}, \ldots, i_{\nu}$, as well as an integer $p$ between 1 and $\nu-1$, such that:

i) The following two matrices are not full-rank:

$$
\begin{aligned}
& {\left[\begin{array}{lllll}
A-\sigma_{i} I_{n} & B_{i_{1}} & B_{i_{2}} & \ldots & B_{i_{p}}
\end{array}\right]} \\
& {\left[\begin{array}{lllll}
A-\sigma_{i} I_{n} & C_{i_{p+1}}^{T} & C_{i_{p+2}}^{T} & \ldots & C_{i_{\nu}}^{T}
\end{array}\right]^{T}}
\end{aligned}
$$

ii) The equality given below holds for any complex number $s \neq \sigma_{j}, j=1,2, \ldots, n$ :

$$
\begin{aligned}
& {\left[\begin{array}{llll}
C_{i_{p+1}}^{T} & C_{i_{p+2}}^{T} & \ldots & C_{i_{\nu}}^{T}
\end{array}\right]^{T}\left(A-s I_{n}\right)^{-1}} \\
& \times\left[\begin{array}{llll}
B_{i_{1}} & B_{i_{2}} & \ldots & B_{i_{p}}
\end{array}\right]=0
\end{aligned}
$$

Proof: The proof follows directly from Theorem 1.

Theorem 2 The SDFMs of the system $\mathcal{S}\left(S_{1}, S_{2}, \ldots, S_{\nu}\right)$ are identical to its QFMs.

Proof: Assume that $\sigma_{i}$ is an SDFM of the system $\mathcal{S}\left(S_{1}, S_{2}, \ldots, S_{\nu}\right)$, and consider the integers $i_{1}, i_{2}, \ldots, i_{\nu}$ in Corollary 1. Define now two new composite subsystems $\mathbf{S}_{1}$ and $\mathbf{S}_{2}$, where $\mathbf{S}_{1}$ is composed of $p$ subsystems $S_{1}, S_{2}, \ldots, S_{p}$, and $\mathbf{S}_{2}$ is composed of $\nu-p$ subsystems $S_{p+1}, S_{p+2}, \ldots, S_{\nu}$. One can easily conclude from (13) and the characteristics of DFM given in Davison and Chang (1990), that $\sigma_{i}$ is a DFM of the system $\mathcal{S}\left(\mathbf{S}_{1}, \mathbf{S}_{2}\right)$.

On the other hand, condition (ii) of Corollary 1 implies that there is no edge form $\mathbf{S}_{1}$ to $\mathbf{S}_{2}$ in the digraph of the system; i.e., the system $\mathcal{S}$ consisting of the two subsystems $\mathbf{S}_{1}$ and $\mathbf{S}_{2}$ is not strongly connected (note that a system consisting of two subsystems is strongly connected if and only if the transfer function matrix from each of its subsystems input to the other subsystems output is not identically zero). Furthermore, since the system $\mathcal{S}$ has already been broken down into the subsystems $\tilde{S}_{1}, \tilde{S}_{2}, \ldots, \tilde{S}_{l}$ (where $l$ denotes the minimum number of strongly connected subgraphs of $\mathcal{G}$, as discussed earlier) which are not strongly connected to each other, it can be easily deduced that there exists a permutation of $\{1,2, \ldots, l\}$ denoted by distinct integers $j_{1}, j_{2}, \ldots, j_{l}$, as well as an integer $\zeta$, such that $\mathbf{S}_{1}=\tilde{S}_{j_{1}} \cup \tilde{S}_{j_{2}} \cup \cdots \cup \tilde{S}_{j_{\zeta}}$ and $\mathbf{S}_{2}=\tilde{S}_{j_{\zeta+1}} \cup \tilde{S}_{j_{\zeta+2}} \cup \cdots \cup \tilde{S}_{j_{l}}$. This implies that any DFM of the system $\mathcal{S}\left(\mathbf{S}_{1}, \mathbf{S}_{2}\right)$ is also a DFM of the system $\mathcal{S}\left(\tilde{S}_{1}, \tilde{S}_{2}, \ldots, \tilde{S}_{l}\right)$. Thus, since it was shown that $\sigma_{i}$ is a DFM of the system $\mathcal{S}\left(\mathbf{S}_{1}, \mathbf{S}_{2}\right)$, it is a DFM of the system $\mathcal{S}\left(\tilde{S}_{1}, \tilde{S}_{2}, \ldots, \tilde{S}_{l}\right)$ as well. On the other hand, it is known from Definition 5 that the DFMs of the system $\mathcal{S}\left(\tilde{S}_{1}, \tilde{S}_{2}, \ldots, \tilde{S}_{l}\right)$ are, in fact, the QFMs of the system $\mathcal{S}\left(S_{1}, S_{2}, \ldots, S_{\nu}\right)$. Therefore, $\sigma_{i}$ is a QFM of the system $\mathcal{S}\left(S_{1}, S_{2}, \ldots, S_{\nu}\right)$.

Assume now that $\lambda$ is a QFM of the system $\mathcal{S}\left(S_{1}, S_{2}, \ldots\right.$, $\left.S_{\nu}\right)$. Hence, $\lambda$ is either an SDFM or a UDFM. If it is a UDFM, then it follows from Lemma 1 that $\lambda$ is not fixed with respect to a discrete-time controller with a $\mathrm{ZOH}$. A well-known property of QFM, however, is that $\lambda$ is fixed with respect to any type of control law (Gong and Aldeen, 1997). This contradicts the original assumption, and hence proves that $\lambda$ is an SDFM of the system $\mathcal{S}\left(S_{1}, S_{2}, \ldots, S_{\nu}\right)$. This completes the proof.

Remark 3 It can be concluded from Conditions (i) and (ii) in Corollary 1 and the discussion in the proof of Theorem 2, that if $\sigma_{i}$ is an SDFM of the system $\mathcal{S}\left(S_{1}, S_{2}, \ldots, S_{\nu}\right)$ (or equivalently a $Q F M$ of the system), then the system can be partitioned into two subsystems $\mathbf{S}_{1}$ and $\mathbf{S}_{2}$ such that $\sigma_{i}$ is an uncontrollable mode of the system $\mathcal{S}$ from the input of the subsystem $\mathbf{S}_{1}$, and an unobservable mode of $\mathcal{S}$ from the output of the subsystem $\mathbf{S}_{2}$. Moreover, the transfer function matrix from the input of $\mathbf{S}_{1}$ to the output of $\mathbf{S}_{2}$ is zero.

The following result follows immediately from Theorem 2 and Lemma 1.

Corollary 2 Assume that the system $\mathcal{S}\left(S_{1}, S_{2}, \ldots, S_{\nu}\right)$ had $P_{s}$ QFMs denoted by $\lambda_{i}, i=1,2, \ldots, P_{s}$, and $P_{u}$ non-quotient DFMs denote by $\bar{\lambda}_{i}, i=1,2, \ldots, P_{u}$. The discrete-time equivalent model $\mathcal{S}_{d}\left(\mathcal{S}_{d_{1}}, \mathcal{S}_{d_{2}}, \ldots, \mathcal{S}_{d_{\nu}}\right)$ has only $P_{s}$ DFMs $e^{\lambda_{i} h}, i=1,2, \ldots, P_{s}$, which correspond to the QFMs of $\mathcal{S}\left(S_{1}, S_{2}, \ldots, S_{\nu}\right)$, for almost all values of $h$. In other words, the DFMs $\bar{\lambda}_{i}, i=1,2, \ldots, P_{u}$, of $\mathcal{S}\left(S_{1}, S_{2}, \ldots, S_{\nu}\right)$ will be eliminated by sampling.

It is shown in Gong and Aldeen (1997) and Anderson and Moore (1981), that a system with no unstable QFMs 
can be stabilized by using an appropriate time-varying decentralized control law. However, as stated in Corollary 2, one can use a sampled-data decentralized controller as a time-varying control law for the continuoustime system. It is to be noted that a discrete-time controller has its unique advantages; moreover, it is easy to design and implement. It is worth noticing that the results obtained in this paper can be easily utilized to design a discrete-time compensator with a generalized sampled-data hold function to achieve a better performance (Rossi and Miller, 1999; Juan and Kabamba, 1991; Lavaei and Aghdam, 2007a,b; Aghdam, Davison, and Arreola, 2006; Aghdam and Davison, 2007).

Remark 4 The present work initially assumes that all of the modes of the system $\mathcal{S}$ are distinct. In order to relax this assumption to some degree, suppose that $\mathcal{S}$ has some repeated modes, none of which is a DFM. Apply first a generic static output feedback decentralized controller to the system to move the repeated modes. The results derived in this paper can then be applied to the resultant closed-loop system. Therefore, those modes that are not $Q F M s$ of the system $\mathcal{S}$ can be displaced via a hybrid controller, comprising a decentralized static continuous-time and a decentralized LTI discrete-time controllers.

Remark 5 Since the notion of structured DFMs was merely defined based on the decoupled realization of the system, $\mathcal{S}$ was initially transformed into a decoupled form. Later on, it was shown that the QFMs of the decoupled system are tantamount to the structured DFMs of the system. Nonetheless, it is important to note that the QFMs of a system are invariant under any similarity transformation. Thus, the QFMs of the system $\mathcal{S}$ are properly characterized by the proposed method, and the decentralized stabilizability results obtained here are independent of the state-space representation of the system.

\section{Illustrative example}

Consider a system $\mathcal{S}$ consisting of three SISO subsystems with the state-space matrices give in (5). Let $i_{j}=j, j=$ $1,2,3$ and $p=1$ in Theorem 1 which yield that $\lambda=1$ is an SDFM of the system $\mathcal{S}\left(S_{1}, S_{2}, S_{3}\right)$. In other words, if the nonzero entries of the vectors $B_{i}, C_{i}, i=1,2,3$ are replaced by any arbitrary numbers, then $\lambda=1$ still remains a DFM of the resultant system. Moreover, it was shown in the paper (by using the structural graph of the system and the schematic depicted in Figure 2) that $\lambda=1$ is a QFM of the system $\mathcal{S}\left(S_{1}, S_{2}, S_{3}\right)$. In other words, $\lambda=1$ is both an SDFM and a QFM of the system $\mathcal{S}\left(S_{1}, S_{2}, S_{3}\right)$. This is in accordance with the result of Theorem 2 .

Now, let the vectors $B_{1}, C_{2}$, and $C_{3}$ in (5) be replaced by the ones given in (7). As pointed out earlier, in this case, $\lambda=1$ is a DFM of the system $\mathcal{S}\left(S_{1}, S_{2}, S_{3}\right)$, but it is not a UDFM; hence, it is not a QFM either (according to Theorem 2). Therefore, this mode can be eliminated by means of sampling according to Corollary 2. For instance, choose $h=1$ sec. It is straightforward to show that the modes of the open-loop discrete-time equivalent model are $0.0498,0.1353,2.7183$, while those of the discrete-time model under a static decentralized feedback with the unity gain are $2.0685 \pm 0.7942 i,-4.9743$. Since these two sets of modes are disjoint, it can be concluded that the discrete-time equivalent model does not have any DFMs, as expected from Corollary 2.

\section{Conclusions}

This paper deals with the decentralized control problem for a broad class of interconnected systems. It is proved that the notions of a structured decentralized fixed mode and a quotient fixed mode introduced in the literature are identical for linear time-invariant, controllable and observable systems with distinct and nonzero eigenvalues. As a result of this equivalency, it is shown that if there exists a decentralized controller with a general structure (e.g., nonlinear or time-varying) to stabilize a system belonging to the aforementioned class, then there also exists a decentralized LTI discrete-time controller (with a zero-order hold), which stabilizes the system. The numerical example confirms the results of the paper.

\section{References}

Aghdam, A. G., Davison, E. J. \& Arreola, R. B. (2006). Structural modification of systems using discretization and generalized sampled-data hold functions. Automatica. 42(11), 1935-1941.

Aghdam, A. G., \& Davison, E. J. (2007). Digital control design for systems with approximate decentralized fixed modes. to appear in Automatica.

Anderson, B. D. O., \& Moore, J. B. (1981). Timevarying feedback laws for decentralized control. IEEE Transactions on Automatic Control. 26(5), 1133-1139.

Chen, T., \& Francis, B. (1995).Optimal sampled-data control systems. Springer.

Davison, E. J., \& Chang, T. N. (1990). Decentralized stabilization and pole assignment for general proper systems. IEEE Transactions on Automatic Control. 35(6), 652-664.

Davison, E. J., \& Wang, S. H. (1973). Properties of linear time-invariant multivariable systems subject to arbitrary output and state feedback. IEEE Transactions on Automatic Control. 18(1), 24-32.

Gong, Z., \& Aldeen, M. (1997). Stabilization of decentralized control systems. Journal of Mathematical Systems, Estimation, and Control. 7(1), 1-16.

Jamshidi, M. (1997). Large-scale systems: Modelling, control and fuzzy logic. Upper Saddle River, NJ: Prentice-Hall. 
Juan, Y., \& Kabamba, P. T. (1989). Simultaneous pole assignment in linear periodic systems using constrained structure feedback. IEEE Transactions on Automatic Control. 34(2), 168-173.

Juan, Y. C., \& Kabamba, P. T. (1991). Optimal hold functions for sampled data regulation. Automatica. 27(1), 177-181.

Khargonekar, P. P., \& Özgüler, A. B. (1994). Decentralized control and periodic feedback. IEEE Transactions on Automatic Control. 39(4), 877-882.

Lavaei, J., \& Aghdam, A. G. (2007a). Optimal periodic feedback design for continuous-time LTI systems with constrained control structure. International Journal of Control. 80(2), 220-230.

Lavaei, J., \& Aghdam, A. G. (2007b). Simultaneous LQ control of a set of LTI systems using constrained generalized sampled-data hold functions. Automatica. 43(2), 274-280.

Özgüner, Ü., \& Davison, E. J. (1985). Sampling and decentralized fixed modes, Proceedings of the 1985 American Control Conference. Boston, Massachusetts (pp. 257-262).

Rossi, M., \& Miller, D. E. (1999). Gain/phase margin improvement using static generalized sampled-data hold functions. Systems \& Control Letters. 37(3), 163-172.

Sezer, M. E., \& Šiljak, D. D. (1981). Structurally fixed modes. Systems \& Control Letters. 1(1), 60-64.

Sezer, M. E., \& Šiljak, D. D. (1990). Decentralized multirate control. IEEE Transactions on Automatic Control. 35(1), 60-65.

Šiljak, D. D. (1991). Decentralized control of complex systems. Cambridge: Academic Press.

Wang, S. H., \& Davison, E. J. (1973). On the stabilization of decentralized control systems. IEEE Transactions on Automatic Control. 18(5), 473-478. 